\title{
Noninvasive Assessment of Blood Pressure Variability in Preterm Infants
}

\author{
PETER ANDRIESSEN, RICK L.M. SCHOFFELEN, RALPH C.M. BERENDSEN, \\ NICOLE A.M. DE BEER, S. GUID OEI, PIETER F.F. WIJN, AND CARLOS E. BLANCO
}

\begin{abstract}
Neonatal Intensive Care Unit [P.A.], Department of Clinical Physics [R.L.M.S., R.C.M.B., P.F.F.W.], and Department of Gynecology and Obstetrics [S.G.O.], Máxima Medical Center, 5500 MB, Veldhoven, The Netherlands; Department of Electrical Engineering [N.A.M.d.B.], and Department of Physics [P.F.F.W.], Eindhoven University of Technology, Den Dolech 2, 5612 AZ, Eindhoven, The Netherlands; Department of Pediatrics [C.E.B.], Division of Neonatology, Academic Hospital Maastricht, 6202 AZ, Maastricht, The Netherlands
\end{abstract}

\section{ABSTRACT}

The feasibility of measuring blood pressure (BP) variability by a noninvasive beat-to-beat finger arterial BP device (Finapres) was assessed in preterm infants. By application of the finger cuff around the infant's wrist, time and frequency domain (spectral power) analysis of noninvasive beat-to-beat BP signals were compared with intra-arterial measurements. A fast Fourier Transform was used to compute the spectral power density from 128-s periods. The low-frequency band (LF; $0.04-0.15 \mathrm{~Hz}$ ) is partly associated with baroreflex activity. The high-frequency band (HF; $0.4-1.5 \mathrm{~Hz}$ ) is associated with respiratory activity. In eight subjects above $1000 \mathrm{~g}$, reliable signals could be obtained. We observed a high correlation between noninvasive and intraarterial beat-to-beat systolic BP values (mean $\mathrm{r}$ value $\pm \mathrm{SD}, 0.87$ \pm 0.11 ), with a gain close to 1 (mean gain $\pm \mathrm{SD}, 1.0 \pm 0.4 \mathrm{~mm}$ $\mathrm{Hg} / \mathrm{mm} \mathrm{Hg}$ ). Finapres estimated beat-to-beat systolic BP changes more accurately than diastolic values. We found a very high amount of linear coupling, expressed as coherence function, between the power spectra of noninvasive and intra-arterial systolic BP measurements. For systolic BP, the (pooled) group mean \pm SEM coherence values were $0.93 \pm 0.00$ and $0.91 \pm$ 0.01 for LF and HF fluctuations, respectively (NS). The wrist method of Finapres in neonates has limited value in estimating absolute BP but is useful in a clinical research situation, where identification of beat-to-beat changes in systolic BP is more important. Finapres provides a noninvasive tool for investigating autonomic cardiovascular regulation (baroreflex sensitivity, spectral analysis of BP fluctuations) in neonates. (Pediatr Res 55: 220-223, 2004)
BP, blood pressure
DBP, diastolic blood pressure
HF, high frequency
LF, low frequency
SBP, systolic blood pressure

Abbreviations
Finapres (FINger Arterial PRESsure) is a noninvasive blood pressure (BP) method that is based on the volume-clamp principle (1). The device is based on a photoplethysmographic system applied to the finger and provides a continuous beatto-beat waveform. Several studies of Finapres have been reported and have shown good agreement with intra-arterial measured BP, in both adults and children (2-4). Despite abundant literature about the application of Finapres in adults, very little is known in neonates. Drouin et al. (5) published in 1997 about the accuracy of Finapres measurements in the neonate. Because no appropriately sized cuff exists for neo-

Received May 9, 2003; accepted August 12, 2003.

Correspondence: Peter Andriessen, Máxima Medical Center, Neonatal Intensive Care Unit, PO Box 7777, 5500 MB, Veldhoven, The Netherlands; e-mail: P.Andriessen@mmc.nl

DOI: 10.1203/01.PDR.0000104152.85296.4F nates, Drouin placed the finger cuff around the wrist of the baby. Absolute Finapres BP values were compared with intraarterial BP for selected periods of $5 \mathrm{~s}$, and it was concluded that both methods gave similar results. However, no information was given about the accuracy of measuring BP fluctuations over longer periods of time. We do know from studies in adults, however, that Finapres may overestimate the lowfrequency (LF) fluctuation (6). The reliability of Finapres to produce frequency domain analysis in neonates has not been assessed. The aim of this study was to determine 1) the accuracy of Finapres in measuring BP absolute values and beat-to-beat changes and 2) the accuracy of Finapres in studying $\mathrm{BP}$ variability by frequency domain analysis.

\section{METHODS}

The study was performed in 12 preterm infants (gestational age, 26-33 wk; birth weight, 750-2215 g) whose intensive 
care management required an umbilical arterial catheter. Informed consent was obtained from the parents of each subject. The local Ethics Committee approved the study.

The Finapres device (model 5, TNO Institute of Applied Physics, Biomedical Instrumentation, Amsterdam, The Netherlands) consists of a finger BP cuff with a built-in infrared sensitive photocell; a pressure manometer and an automatic unit for cuff inflation; and a monitor with digital display of systolic BP (SBP) and diastolic BP (DBP), and heart rate. After self-calibration, a servomechanism pneumatically attached to a noncompliant cuff that fits around the finger continuously adjusts air pressure in the cuff to maintain a constant transmural pressure in the digital arteries. This is achieved by continuously monitoring a photoelectric signal that passes through the finger. Absolute pressure values are determined at the time of self-calibration by a proprietary algorithm. After calibration, the servosystem tries to nullify the pulsations. When this occurs, the transmural pressure is zero and an approximately linear relation exists between intra-arterial pressure and transmittance (2).

Arterial BP was measured invasively through a 4-French catheter placed earlier in the lower aortic position for routine monitoring of vital functions and intensive care management. A 0.5 $\mathrm{mL} / \mathrm{h}$ infusion of heparinized physiologic saline solution was continuously flushed through the catheter. The smallest (white) cuff supplied with the Finapres device was placed around the left wrist. After self-calibration (Physiocal), 10-min recordings (servo control off) were obtained in each subject. During the procedure of Finapres measurement, the skin colored temporarily dark red to blue during the insufflation of the cuff, which restored to normal immediately after desufflation of the cuff. None of the infants sustained vascular damage or discomfort from the use of the wrist cuff during the measurements.

For each subject, we selected a period of $128 \mathrm{~s}$ (consisting of three half-overlapping segments of $64 \mathrm{~s}$ ) for further data analysis. A 2-min period was chosen as a compromise between the need for signal stability and risk on movement artifacts from the wrist cuff.

Both BP signals were sampled at $128 \mathrm{~Hz}$ and stored on a data acquisition system. SBP and DBP were identified from "peak" detection of the BP signal in a 128-s period. This procedure resulted in an unevenly spaced "systogram" and "diastogram." These unevenly time-spaced series were resampled into equidistantly spaced series using a BoxCar window at $4 \mathrm{~Hz}$ (7). Spectral leakage was reduced by subtracting the DC variability and by multiplying with a triangular Parzen windowing (8). Filter correction removed unwanted side effects of resampling and Parzen windowing. Each 128-s period of preprocessed evenly spaced BP series was subdivided in three half-overlapping 64-s (256 points) segments. A Fast Fourier Transform was used to compute the power spectral density. A mean power spectrum was derived from three half-overlapping 64-s spectral density functions to increase accuracy. The total power was computed for the frequency band between 0.04 and $1.5 \mathrm{~Hz}$. The value for power was expressed in $\mathrm{mm} \mathrm{Hg}^{2}$. The LF band, partly associated with baroreflex activity, was defined between 0.04 and $0.15 \mathrm{~Hz}$. The HF band, associated with respiratory activity, was defined between 0.4 and $1.5 \mathrm{~Hz}(9)$. The maximum (Nyquist) frequency is $1.5 \mathrm{~Hz}$, assuming the heart rate to be $<3 \mathrm{~Hz}$ or $180 \mathrm{bpm}$.
Statistical analysis. Bias in absolute and beat-to-beat SBP and DBP values was calculated as the mean of difference between noninvasive and intra-arterial BP values (bias), with its $95 \%$ limits of agreement (10). The SD of the bias in these signals was calculated in each patient as an index of intrapatient variation. BP gain and offset were calculated by linear regression between noninvasive and arterial values of SBP and DBP: (beat-to-beat) $\mathrm{BP}_{\text {noninvasive }}=$ gain * (beat-to-beat) $\mathrm{BP}_{\text {invasive }}+$ offset (11).

In addition, we estimated, in frequency domain, the amount of coupling (squared coherence function) between the two power spectra of Finapres and intra-arterial BP. The coherence function is comparable to regression analysis in the time domain, except that a correlation is computed for each frequency region (12). The coherence function ranges between 0 (no relation) and 1 (a perfect relation). With respect to details of data analysis and transfer function (coherence), we refer to an earlier report of our group (13). The spectral resolution was $0.016 \mathrm{~Hz}$ (1/64 s), yielding seven (coherence) values for the LF $(0.04-0.15 \mathrm{~Hz})$ band and 70 values for the HF $(0.4-1.5 \mathrm{~Hz})$ band. In each subject, a mean coherence $\pm \mathrm{SD}$ was computed for the LF and HF band and was used to assess the statistical reliability of the two power spectra. Pooled (group) results of the coherence values are presented as mean $\pm \mathrm{SE}$ of measurements (SEM).

Comparisons between SBP and DBP values or between LF and HF values were made with a (two-sided) paired $t$ test for parametric data. Statistical significance was accepted at $p<0.05$.

\section{RESULTS}

Reliable Finapres waveform signals could be obtained in eight (gestational age range,: 26.1-33.4 wk; weight range, $1000-2280 \mathrm{~g}$ ) of 12 subjects. All failures were due to bad fitting of the cuff around the small wrist in infants with a weight $<1000 \mathrm{~g}$. All but one infant were circulatory stable at the time of measurement, and echocardiography in these subjects revealed no structural abnormalities. One infant (subject 7) with a patent ductus arteriosus required vasoactive drugs to maintain adequate BP.

A total of 2502 paired BP measurements were analyzed, yielding a mean of 313 analyzed beats per selected 128-s period per subject (range, 300-345). A representative tracing of beat-to-beat SBP values obtained in subject 1 is shown in Figure 1.

The mean intra-arterial BP was $55.6 \pm 10.2 \mathrm{~mm} \mathrm{Hg}$ systolic and $30.0 \pm 5.5 \mathrm{~mm} \mathrm{Hg}$ diastolic in the study group. Table 1 shows the individual results of comparing absolute BP values. We observed considerable variation in under- and overestimation of the intra-arterial pressure values between subjects. Bias ranged from -6.5 to $17.6 \mathrm{~mm} \mathrm{Hg}$ systolic and from -5.6 to $17.9 \mathrm{~mm} \mathrm{Hg}$ diastolic. Details of the individual 95\% limits of agreement are shown in Table 1 . The mean correlation coefficients were $0.78 \pm 0.18$ systolic and $0.80 \pm 0.11$ diastolic (NS). The mean gains of absolute BP measurements were 1.0 $\pm 0.3 \mathrm{~mm} \mathrm{Hg} / \mathrm{mm} \mathrm{Hg}$ systolic and $0.8 \pm 0.2 \mathrm{~mm} \mathrm{Hg} / \mathrm{mm} \mathrm{Hg}$ diastolic (NS), with offsets of $3.2 \pm 12.9$ and $7.7 \pm 6.9 \mathrm{~mm}$ $\mathrm{Hg}$, respectively (NS). 


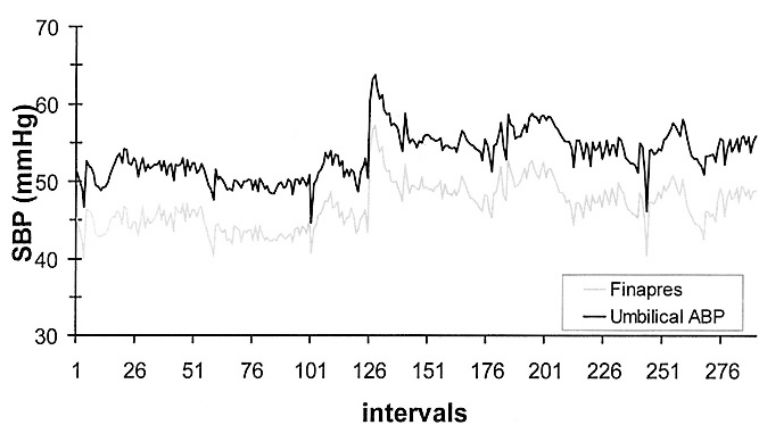

Figure 1. Tracing of SBP values obtained in a preterm infant of $1260 \mathrm{~g}$ (subject 1). This tracing shows beat-to-beat intra-arterial (black line) and Finapres (gray line) SBP values of a selected period of $128 \mathrm{~s}$. Note the difference (bias) in SBP values (approximately $6.5 \mathrm{~mm} \mathrm{Hg}$ ) between both methods, but with accurate tracking, the BP changes.

Table 1. Results of absolute BP values obtained by both methods of the study group

\begin{tabular}{crrrcc}
\hline & \multicolumn{2}{c}{ Bias } & & \multicolumn{2}{c}{$95 \%$ Limits of agreement } \\
\cline { 2 - 3 } \cline { 5 - 6 } Subject & SBP & DBP & & SBP & DBP \\
\hline 1 & -6.5 & -3.5 & & -8.1 to -5.0 & -5.7 to -1.3 \\
2 & -5.7 & 1.7 & & -7.3 to -4.1 & 0.9 to 2.5 \\
3 & -4.5 & 2.1 & & -6.3 to -2.7 & 0.3 to 3.9 \\
4 & 4.4 & 17.9 & & -1.4 to 10.2 & 14.7 to 21.1 \\
5 & 7.0 & 4.9 & & 3.4 to 10.6 & 2.7 to 7.1 \\
6 & 5.2 & 7.9 & & 3.4 to 7.0 & 6.7 to 9.1 \\
7 & 17.6 & -5.6 & & 13.6 to 21.6 & -9.4 to -1.8 \\
8 & 0.3 & -4.5 & & -1.9 to 2.5 & -5.7 to -3.3 \\
\hline
\end{tabular}

Bias (mean of the difference) and 95\% limits of agreement for SBP and DBP, respectively. Bias and $95 \%$ limits of agreement are expressed in $\mathrm{mm} \mathrm{Hg}$.

In Table 2, the beat-to-beat changes of Finapres and arterial BP signals are presented. Remarkably, no bias existed between both methods for the beat-to-beat BP values. Details of the individual 95\% limits of agreement are shown in Table 2. The mean correlation coefficients were $0.87 \pm 0.11$ systolic and $0.76 \pm 0.22$ diastolic $(p=0.05)$. The mean gains of beat-tobeat BP measurements were $1.0 \pm 0.4 \mathrm{~mm} \mathrm{Hg} / \mathrm{mm} \mathrm{Hg}$ systolic and $0.8 \pm 0.2$ diastolic $(p<0.05)$, with offsets of $0.0 \pm 0.0$ and $0.0 \pm 0.0 \mathrm{~mm} \mathrm{Hg}$, respectively (NS). The diastolic beat-to-beat gain $(<1)$ indicates increasing negative bias with increasing beatto-beat BP changes.

Figure 2 illustrates the power spectra of intra-arterial and Finapres systolic signals in subject 1 . Note that the two spectra

Table 2. Results of beat-to-beat changes between arterial BP and Finapres signals

\begin{tabular}{cccccc}
\hline & \multicolumn{2}{c}{ Bias } & & \multicolumn{2}{c}{$95 \%$ Limits of agreement } \\
\cline { 2 - 3 } Subject & SBP & DBP & & SBP & DBP \\
\hline 1 & 0.0 & 0.0 & -1.2 to 1.2 & -1.4 to 1.4 \\
2 & 0.0 & 0.0 & -0.8 to 0.8 & -0.7 to 0.7 \\
3 & 0.0 & 0.0 & -2.1 to 2.1 & -2.0 to 2.0 \\
4 & 0.0 & 0.0 & -3.1 to 3.1 & -1.9 to 1.9 \\
5 & 0.0 & 0.0 & -2.1 to 2.1 & -1.9 to 1.9 \\
6 & 0.0 & 0.0 & -1.7 to 1.7 & -1.1 to 1.1 \\
7 & 0.0 & 0.0 & -3.3 to 3.3 & -2.2 to 2.2 \\
8 & 0.0 & 0.0 & -1.7 to 1.7 & -1.4 to 1.4 \\
\hline
\end{tabular}

Bias (mean of the difference) and 95\% limits of agreement for SBP and DBP beat-to-beat changes, respectively. Bias and $95 \%$ limits of agreement are expressed in $\mathrm{mm} \mathrm{Hg}$.

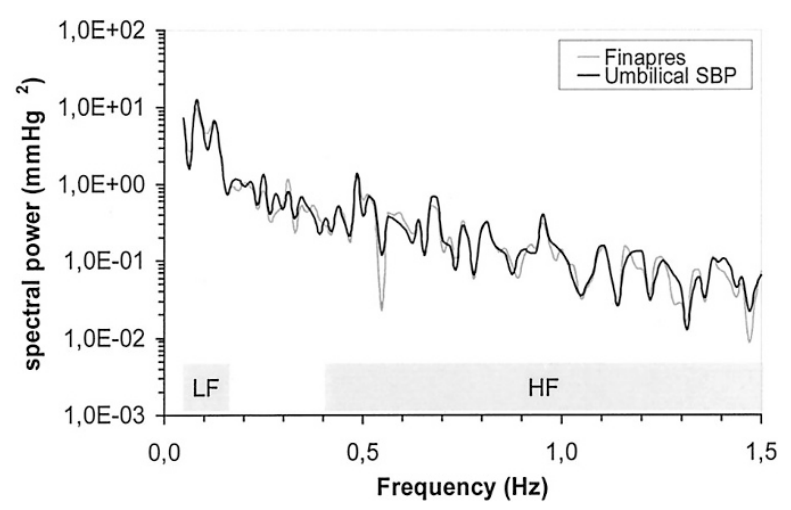

Figure 2. Spectral power curves obtained by both methods of the same infant as in Figure 1. In this figure, two power spectra of intra-arterial SBP (black line) and Finapres (gray line) measurements are shown of the SBP data. The LF band was defined between 0.04 and $0.15 \mathrm{~Hz}$. The HF band was defined between 0.4 and $1.5 \mathrm{~Hz}$. Note that the two power spectra look similar. Spectral power (logarithmic scale) was expressed in $\mathrm{mm} \mathrm{Hg}^{2}$. In this subject, the mean coherence value for LF was $0.97 \pm 0.03$ and for HF was $0.94 \pm 0.06$.

look similar. In this subject, the mean coherence value for LF was $0.97 \pm 0.03$ and for HF was $0.94 \pm 0.06$. In Table 3 , the mean coherence values for the LF and HF bands are shown for the total group. For LF fluctuations, the (pooled) group mean \pm SEM coherence values were $0.93 \pm 0.00$ systolic and $0.91 \pm 0.01$ diastolic (NS). For HF fluctuations, the mean coherence values were $0.91 \pm 0.01$ systolic and $0.82 \pm 0.02$ diastolic $(p=0.07$; NS). For SBP, no significant difference was found in coherence values between LF and HF. For DBP, a significant difference in coherence values was found between LF and HF $(p<0.05)$.

\section{DISCUSSION}

We found considerable differences between the noninvasive Finapres technique and umbilical arterial BP measurements for absolute BP values, with a great variation in bias and limits of agreement in the study group. In contrast, we found a good correlation between beat-to-beat values and no bias between both methods, indicating accurate estimation of beat-to-beat changes of BP values. In addition, we found high coherence values between noninvasive and intra-arterial BP power spectra in both HF and LF bands.

The only available study in neonates, using the same wrist position, reported comparable results with the $95 \%$ limits of agreement -5.3 to $8.7 \mathrm{~mm} \mathrm{Hg}$ for SBP and -5.2 to $5.5 \mathrm{~mm} \mathrm{Hg}$ for DBP (5). Their conclusion of "both methods gave similar results" needs further clarification, in our opinion. In contrast to our method of analyzing a complete 2-min period, they analyzed five very short periods of $5 \mathrm{~s}$, resulting in a limited amount of BP values. From Table 1, it is clear that there is considerable variation in bias among the subjects. It is therefore questionable whether one may pool all individual BP values, as done in the former study. In addition, we can expect more pronounced differences between both methods in our population than in adults, because we compare lower aortic pressure with "radial" BP. More distal in the arterial tree, the BP waveform generally shows overshoot of the systolic BP. Because this overshoot depends on characteristics of the arterial tree, which may vary substantially between subjects, Finapres is not reliable in estimating absolute BP values. Like 
Table 3. Coherence values of the LF $(0.04-0.15 \mathrm{~Hz})$ and $\mathrm{HF}(0.4-1.5 \mathrm{~Hz})$ bands for SBP and DBP

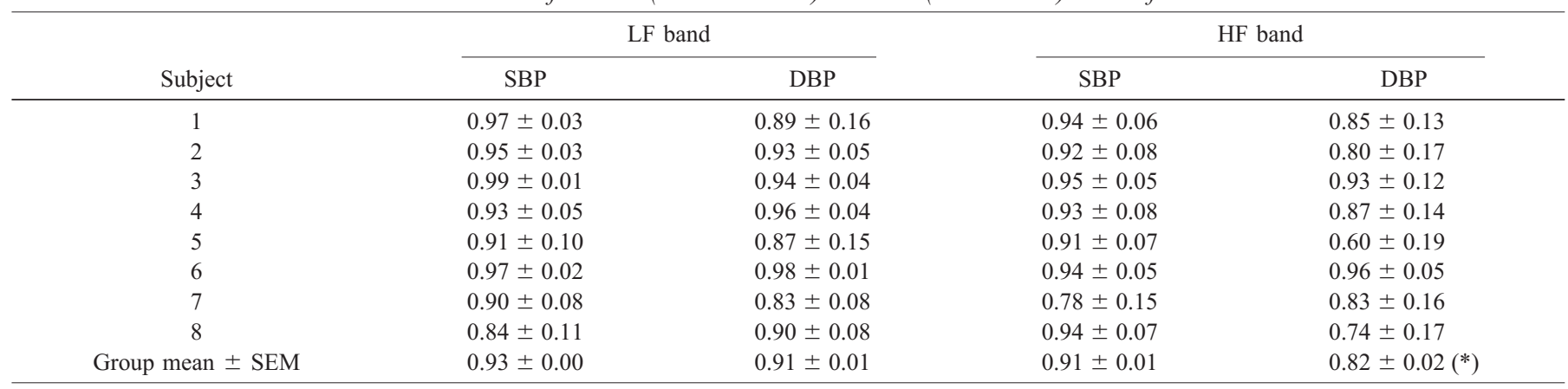

The mean $\pm \mathrm{SD}$ of the coherence values for the LF $(0.04-0.15 \mathrm{~Hz})$ and HF $(0.4-1.5 \mathrm{~Hz})$ bands are shown for SBP and DBP, respectively. At the bottom, the group mean \pm SEM are presented. No significant differences in coherence values other than $*(p<0.05)$, LF versus HF for DBP only.

others, we observed substantial variation in under- and overestimation of the intra-arterial pressure values between subjects (11, 14). Vasoconstriction in relation to temperature seems unlikely because the incubator preserves a thermoneutral environment. In addition, variation might be related to cuff fitting as a result of the small size of the wrist or tissue edema. The used physiocal algorithm for servo calibration was not developed for cuff application at the wrist. Therefore, the algorithm does not always succeed in finding the correct cuff pressure for vascular unloading. The averaged SD of the difference between both methods was low for SBP $(1.4 \pm 0.8)$ and DBP $(1.0 \pm 0.5)$, indicating consistency of measurement for individual subjects. In four subjects with birth weight $<1000 \mathrm{~g}$, no reliable signals could be obtained because of the very small size of the wrist.

In pediatric patients, it is concluded that although substantial measurement bias exists between the noninvasive technique and intra-arterial BP measurements, frequency response analysis suggests that noninvasive BP measurement accurately follows BP changes (11). Also, we found a very good correlation between beat-to-beat values between both methods indicating accurate estimation of beat-to-beat changes of particularly SBP values. The $\mathrm{SD}$ of successive beat-to-beat changes in heart rate or BP within a certain period provides a rough estimate of short-term variability. The (time-domain) short-term variability is similar to the (frequency-domain) HF spectral peak, associated with respiratory activity (9). For systolic beat-to-beat measurements, we observed a higher correlation than for diastolic values. The gain close to 1 and offset of 0 for systolic beat-to-beat values indicates no systematic error in over- or underestimation of intra-arterial systolic measurements. The gain for diastolic (beat-to-beat) values (mean approximately 0.8 ), however, indicates increasing negative bias with increasing beat-to-beat BP changes. These findings suggest that Finapres estimates systolic changes more accurately than diastolic changes.

In addition, we found very high coherence values between arterial and Finapres BP power spectra in both HF and LF bands. This strongly indicates a high amount of linear coupling between the two power spectra of Finapres and intra-arterial BP. No differences were found between LF and HF bands for systolic values, indicating that no overestimation was present for the LF fluctuations as seen in adults (6). Although it did not reach statistical significance, we observed a tendency for a higher amount of linear coupling in the HF band for SBP than for DBP measurements.

\section{CONCLUSION}

In conclusion, this study demonstrates that Finapres estimates SBP changes accurately and estimates BP power spectra reliably. Because baroreflex sensitivity specifies the ratio between changes in R-R interval time and changes in SBP $(\mathrm{ms} / \mathrm{mm} \mathrm{Hg})$, Finapres could be useful in clinical research to quantify this measure. Spontaneous baroreflex sensitivity has been assessed using (the wrist method) Finapres in neonates by time-domain analysis (15). In addition, this noninvasive technique enables use of cross-spectral analysis (transfer function analysis) of heart rate and BP fluctuations (13). Finapres thus provides a noninvasive tool for investigating the autonomic cardiovascular regulation in neonates.

\section{REFERENCES}

1. Penaz J, Voigt A, Teichmann W 1976 Beitrag zur fortlaufenden indirekten Blutdruckmessung. Z Gesamte Inn Med 31:1030-1033

2. Wesseling KH, Settels JJ, de Wit B 1986 The measurement of continuous finger arterial pressure noninvasively in stationary subjects. In: Schmidt TH, Dembroski TM, Bluemchen G (eds) Biological and Psychological Factors in Cardiovascular Disease. Springer-Verlag, Berlin, pp 355-375

3. Parati G, Casadei R, Groppelli A, di Rienzo M, Mancia G 1989 Comparison of finger and intra-arterial blood pressure monitoring at rest and during laboratory testing. Hypertension 13:647-655

4. Tanaka H, Thulesius O, Yamaguchi, Mino M, Konishi K 1994 Continuous noninvasive finger blood pressure monitoring in children. Acta Paediatr 83:646-652

5. Drouin E, Gournay V, Calamel J, Mouzard A, Rozé JC 1997 Feasibility of using finger arterial pressure in neonates. Arch Dis Child Fetal Neonatal Ed 77:F139-F140

6. Omboni S, Parati G, Frattola A, Mutti E, Di Rienzo M, Castiglioni P, Mancia G 1993 Spectral and sequence analysis of finger blood pressure variability. Comparison with analysis of intra-arterial recordings. Hypertension 22:26-33

7. Jaffe RS, Fung DL 1994 Constructing a heart rate variability analysis system. J Clin Monit 10:45-58

8. Akselrod S 1995 Components of heart rate variability: basic studies. In: Malik M, Camm AJ (eds) Heart Rate Variability. Futura Publishing Company, New York, pp $147-163$

9. Rosenstock EG, Cassuto Y, Zmora E 1999 Heart rate variability in the neonate and infant: analytical methods, physiological and clinical observations. Acta Paediatr $88: 477-482$

10. Bland JM, Altman DG 1986 Statistical methods for assessing agreement between two methods of clinical measurement. Lancet 1:307-310

11. Triedman JK, Saul JP 1994 Comparison of intra arterial with continuous non-invasive blood pressure measurements in postoperative pediatric patients. J Clin Monit 10:11-20

12. de Boer RW, Karemaker JM, Strackee J 1985 Relationships between short-term blood pressure fluctuations and heart rate variability in resting subjects: a spectral approach. Med Biol Eng Comput 23:352-358

13. Andriessen P, Koolen AMP, Berendsen RCM, Wijn PFF, ten Broeke EDM, Oei SG, Blanco CE 2003 Cardiovascular fluctuations and transfer function analysis in stable preterm infants. Pediatr Res 53:89-97

14. Jones RDM, Brown AG, Roulson CJ, Smith ID, Chan SC 1992 The upgraded Finapres 2300e: a clinical evaluation of a continuous noninvasive blood pressure monitor. Anaesthesia 47:701-705

15. Drouin E, Gournay V, Calamel J, Mouzard A, Rozé JC 1997 Assessment of spontaneous baroreflex sensitivity in neonates. Arch Dis Child Fetal Neonatal Ed 76:F108-F112 